\title{
Elastic-Plastic Stresses in Shrink Fit with A Solid Shaft
}

\author{
Amal Mouâa ${ }^{1}$, Nor Eddine Laghzale ${ }^{2}$, Abdel-Hakim Bouzid ${ }^{\mathbf{3}}$ \\ 1. LAMAT Laboratory, ENSET, Rabat, Morocco. amal.mouaa@um5s.net.ma \\ 2. LAMAT Laboratory, ENSET, Rabat, Morocco.n.laghzale@um5s.net.ma \\ 3. ASME Fellow, ETS, Montreal, Quebec, Canada. hakim.bouzid@etsmtl.ca
}

\begin{abstract}
Shrink fit joining a solid shaft and a cylinder requires an accurate estimate of the residual contact pressure to transmit high powers, as in the case of gas turbines. Such torques require deformations of materials beyond their elastic limits.

This paper presents an analytical model that analyses the stresses in a shrink fit assembly consisting of a solid shaft and a cylindrical hub operating in the elastic-plastic range. Assuming the hub to have a nonlinear work-hardening material behavior, the distribution of stresses as functions of the interference, and the effect of geometry on contact pressure and on interference are exhibited. To validate the analytic method, Finite Element Method was used.
\end{abstract}

Keywords : shrink fit; solid shaft; elastic-plastic deformation; stresses

\section{Nomenclature:}

$R_{i}, R_{o}$ : Inner and outer hub radii (mm)

$r_{o}$ : Outer solid shaft radius $(\mathrm{mm})$;

$R_{c}$ : Hub elastic-plastic interface radius $(\mathrm{mm})$;

$Y=R_{o} / R_{i}:$ Inner to outer radius ratio; $\sigma_{y}:$ Yield stress $(\mathrm{MPa})$

$Y_{p}=R_{c} / R_{i}:$ Inner to elastic-plastic interface radius ratio

$Y_{e}=R_{o} / R_{c}$ : Elastic-plastic interface to outer radius ratio

$P_{c}$ : Contact pressure to cause partial yielding of hub (MPa)

$I_{c}$ : Interference causing partial yielding of hub $(\mathrm{mm})$

$m$ : Strain hardening exponent

\section{Introduction}

Shrink fit is a technique of joining together two mechanical components usually a hollow or solid shaft and a cylinder. This article is devoted to an assembly with solid shaft, since it is more favored thanks to its ability to maintain the state of stress in the elastic domain. It is also widely used in different mechanical engineering applications such as aircraft engines and aerospace turbopumps. There are few works interested in the study of plasticity of shrink fit assembly with solid shaft in these last twenty years.

Researchers started with thin cylinder theory and assumed a linear strain hardening behavior of the ring material, namely Gamer and Lance in [1] developed an analytic solution of stresses and displacements as a function of the interference in an elastic-plastic shrink fit with hollow shaft. Gamer showed that the yield condition changes for larger values of the interference [2].

Most of the literature is interested in the stresses in the plastic domain of another geometries, for instance, Burenin et al. [3] presented a problem of a shrink fit of a clutch on a shaft, that takes into account the dependence of the yield material strength on the temperature.

An interference fit assembly of a solid shaft and a hub was modelled when interference range remains constant in the work of Shun Wang et al. [4], to investigate how the structure of the assembly influences its contact stress, contact stiffness, and stress concentration.

The last research viewed is the recently published work of Laghzale and Bouzid [5]. It presents an analytical model to analyze the distribution of residual stresses in an interference fit assembly composed of two-hollow cylinders. They obtained the relationship that binds the pressure to the interference using strain hardening parameters.

This paper presents an analytical methodology to analyze the stresses in a shrink fit constituting an elastic solid shaft and elastic-plastic cylindrical hub. The plane strain condition is applied in conjunction with the von Mises yield criteria. In addition, it is assumed that the hub material follows a power law hardening behavior.

\section{Analysis model of the elastic-plastic shrink fit joint}

The developed model is based on Hencky deformation theory and the Von Mises yield criteria. It is supposed that the interference is relatively big to assume that the cylindrical hub undergoes partial or full plasticity. Depending on the maximum level of the contact pressure, the geometry and the material properties of the joint, interference that causes partial yielding of the hub is the case to be considered.

The stress-strain relationship in the plastic range is given by Ludwick equation[6]: $\sigma_{e}=\sigma_{y}+K \varepsilon_{e}^{m}$ for $\sigma_{e} \geq \sigma_{y}(1)$

The Von Mises equivalent stress and strain are as follows:

$$
\begin{gathered}
\sigma_{e}=\sqrt{\frac{1}{2}\left[\left(\sigma_{r}-\sigma_{\theta}\right)^{2}+\left(\sigma_{\theta}-\sigma_{z}\right)^{2}+\left(\sigma_{z}-\sigma_{\theta}\right)^{2}\right]} \\
\varepsilon_{e}=\frac{\sqrt{2}}{3} \sqrt{\left[\left(\varepsilon_{r}-\varepsilon_{\theta}\right)^{2}+\left(\varepsilon_{\theta}-\varepsilon_{z}\right)^{2}+\left(\varepsilon_{z}-\varepsilon_{\theta}\right)^{2}\right]}
\end{gathered}
$$


The volumetric strain equation under plastic condition is given by $\quad \varepsilon_{r}+\varepsilon_{\theta}+\varepsilon_{z}=0$

Under plan strain condition, the axial strain vanishes, and the following is obtained: $\quad \varepsilon_{r}=-\varepsilon_{\theta}$

The radial and tangential strains in cylindrical coordinates for an axisymmetric case are :

$$
\varepsilon_{r}=\frac{d u_{r}}{d r} \quad \text { (6) } \quad ; \quad \varepsilon_{\theta}=\frac{u_{r}}{r}
$$

The equilibrium equation of an axisymmetric body with no variation in the axial direction is :

$$
\frac{d \sigma_{r}}{d r}+\frac{\sigma_{r}-\sigma_{\theta}}{r}=0
$$

Knowing from [5]: $\varepsilon_{e}=\frac{2}{\sqrt{3}} \frac{C}{r^{2}}$ (9); $\frac{d \sigma_{r}}{d r}=\frac{2}{\sqrt{3}} \frac{\sigma_{e}}{r}$

And substituting Eq. (1) into Eq. (11) and integrating between the inside radius $R_{i}$ and intermediate radius $r$ of the hub gives: $\int_{R_{i}}^{r} d \sigma_{r}=\int_{R_{i}}^{r} \frac{2}{\sqrt{3}}\left(\sigma_{y}+K \varepsilon_{e}^{m}\right) \frac{d r}{r}$

\section{Stresses for different cases}

The interference values affect the assembly behavior, as is shown in the work of Laghzale and Bouzid [5]. If we consider $I_{y}$ the interference for which the hub starts to yield at the inner radius, the state of deformation that the assembly undergoes will be according to the two specified conditions below.

\subsection{Interference fit assembly in the elastic range}

The expressions of the radial and circumferential stresses of the hub are known as Lamé's equations.

It is known that the hydrostatic stress state allows the solid shaft to remain elastic. Thus, the radial and circumferential stresses in the solid shaft are constant

$$
\sigma_{\theta_{S}}(r)=\sigma_{r_{S}}(r)=-P
$$

The radial displacement of the shaft considered in plane

$$
\text { strain is : } \quad u_{s}(r)=-P \frac{\left(1+v_{s}\right)\left(1-2 v_{s}\right)}{E_{s}} r
$$

Using the compatibility that equates the interference $I$ to the algebraic summation of the radial displacement of the hub and that of the shaft Eq. (13) at the interface radius $R_{i}$ gives the expression of the contact pressure $P$ such that:

$$
P=I / L
$$

Where $L=\frac{\left(1+v_{s}\right)\left(1-2 v_{s}\right)}{E_{s}} r_{o}+\frac{1+v_{h}}{E_{h}} \frac{R_{i}}{Y^{2}-1}\left(1-2 v_{h}+Y^{2}\right)$

\subsection{Partial plastic deformation of the hub}

When the interference $I \geq I_{y}$, the plastic deformation of the assembly is inevitable. The beginning of plastic behavior of the hub starts at the inner radius, where the equivalent stress reaches the yield stress. Therefore, the pressure required to reach yield is :

$$
P_{y}=\frac{I_{y}}{L}=\frac{\sigma_{y_{h}}\left(Y^{2}-1\right)}{\sqrt{3} Y^{2}}
$$

Let's consider the hub interface radius $r=R_{c}$, beyond which the plastic region ends, as shown in Fig. 1. This case can't be established unless $I \geq I_{y}$.

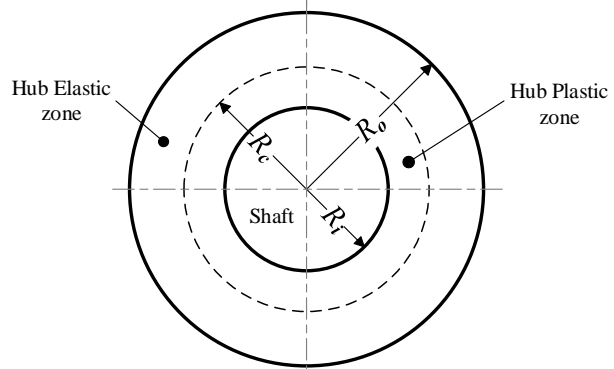

Fig. 1: Assembly posterior to shrink-fit.

To determine the expression of radial stress, Eq. (9) is substituted in Eq, (11) which in turns is integrated between $R_{i}$ and $r$, noting that at $R_{i}$ the radial stress is equal to the contact pressure such that:

$\sigma_{r_{h}}(r)=-P+\frac{2}{\sqrt{3}}\left(\sigma_{y_{h}} \ln \left(\frac{r}{R_{i}}\right)+\frac{K}{2 m}\left(\frac{2 C}{\sqrt{3}}\right)^{m}\left(R_{i}^{-2 m}-r^{-2 m}\right)\right)$

Using Eq. (8) and substituting for the radial stress the tangential stress is :

$$
\begin{aligned}
\sigma_{\theta_{h}}(r)=-P+\frac{2}{\sqrt{3}}\left(\sigma_{y_{h}}\left(\ln \left(\frac{r}{R_{i}}\right)+1\right)+\right. \\
\left.\frac{K}{2 m}\left(\frac{2 C}{\sqrt{3}}\right)^{m}\left(R_{i}^{-2 m}+(2 m-1) r^{-2 m}\right)\right)
\end{aligned}
$$

In the case of plane strain, the hub axial stress is :

$$
\left\{\begin{array}{lll}
\sigma_{z}=v_{h}\left(\sigma_{\theta}+\sigma_{r}\right) & \text { for } & \sigma_{e} \leq \sigma_{y_{h}} \\
\sigma_{z}=\frac{1}{2}\left(\sigma_{\theta}+\sigma_{r}\right) & \text { for } & \sigma_{e} \geq \sigma_{y_{h}}
\end{array}\right.
$$

The constant $C$ is determined by the condition that yielding is reached at the interface radius $R_{c}$ and therefore, substituting $\sigma_{e}=E \varepsilon_{e}$ into Eq. (10) at $r=R_{c}$ and noting that $\sigma_{e}=\sigma_{y_{h}}$ gives: $\quad C=\frac{\sqrt{3}}{2} \frac{\sigma_{y_{h}}}{E_{h}} R_{c}^{2}$

Substituting Eq. (21) into Eq. (17) and noting that the radial stress at $r=R_{c}$ is equal to the pressure required to start yield in the elastic zone per Eq. (16) gives the contact pressure as:

$$
P_{c}=\frac{\sigma_{y_{h}}\left(Y_{e}^{2}-1\right)}{\sqrt{3} Y_{e}^{2}}+\frac{2}{\sqrt{3}}\left(\sigma_{y_{h}} \ln \left(Y_{p}\right)+\frac{K}{2 m}\left(\frac{\sigma_{y_{h}}}{E_{h}}\right)^{m}\left(Y_{p}^{2 m}-1\right)\right)
$$


Using the compatibility of displacements at the interface gives the interference required to yield the hub to the interface radius $R_{c}$

$$
\begin{array}{r}
\mathrm{I}_{c}=\frac{\sqrt{3}}{2} \frac{\sigma_{y_{h}}}{E_{h}} \frac{R_{c}^{2}}{R_{i}}+\frac{\left(1+v_{s}\right)\left(1-2 v_{s}\right) r_{o}}{E_{s}}\left[\frac{\sigma_{y_{h}}}{\sqrt{3}} \frac{Y_{e}^{2}-1}{Y_{e}^{2}}+\right. \\
\left.\quad \frac{2}{\sqrt{3}}\left(\sigma_{y_{h}} \ln \left(Y_{p}\right)+\frac{K}{2 m}\left(\frac{\sigma_{y_{h}}}{E_{h}}\right)^{m}\left(Y_{p}^{2 m}-1\right)\right)\right]
\end{array}
$$

The radial, circumferential and axial stresses in the hub are calculated using Eqs. (17)-(18)-(19)-(20) with the appropriate contact pressure $P$ and constant $C$ in the plastic zone.

\section{Result and discussion}

The present analytical model (AM) allows the evaluation of the radial, circumferential and axial stresses for a precise value of the interference. This model is validated using Finite Element Method (FEM) under ANSYS APDL software. The mechanical characteristics of the solid shaft and the hub are taken from [6] to validate the analytical model.

Using $I_{y}=0.082 \mathrm{~mm}$ and $I_{c}=0.2565 \mathrm{~mm}$, the stresses for elastic-plastic behavior associated to $I_{y} \leq I_{c}$, are presented in Fig. 2. It can be seen from the curves of this graph that the analytical and Finite Element model results superpose each other indicating a good agreement between the two methods.

It is clear from these graphs that the slope of radial stress beyond the elastic-plastic interface decreases throughout the elastic zone until it vanishes at the outer radius. The transition from plastic to elastic zone is visible in the circumferential and axial stresses, they increase gradually in the elastic zone till reaching a maximum value at the elastic-plastic interface and then, tangential stress decreases in the elastic zone and axial stress drops to a constant value.

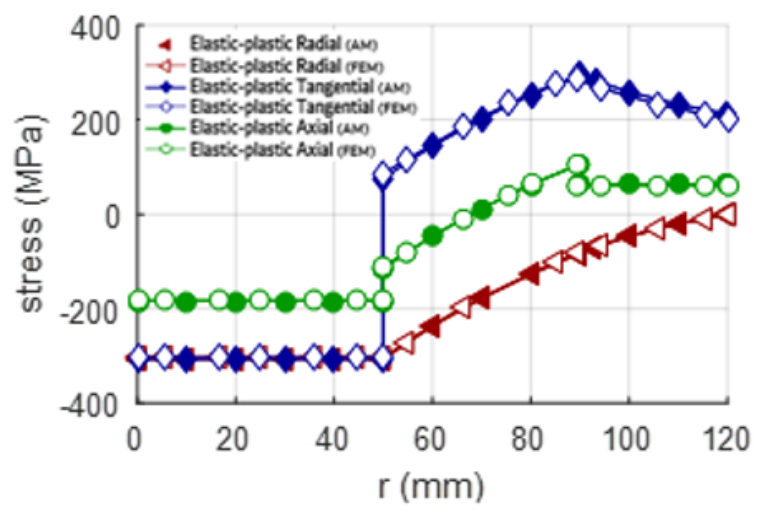

Fig.2: Stresses in the elastic-plastic range.

With the elastic behavior radius ratio does not affect the interference value, the pressure is almost invariable too. Quite the contrary, with elastic-plastic behavior, the increase of the ratio leads to a rise in pressure and interference value.

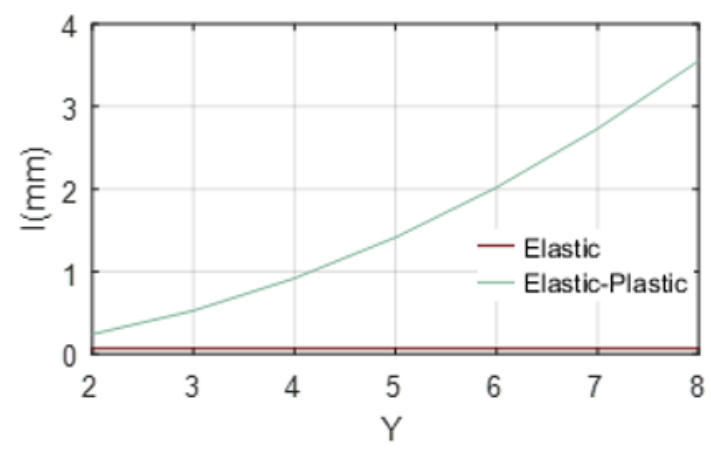

Fig. 3: Interference over radius ratio

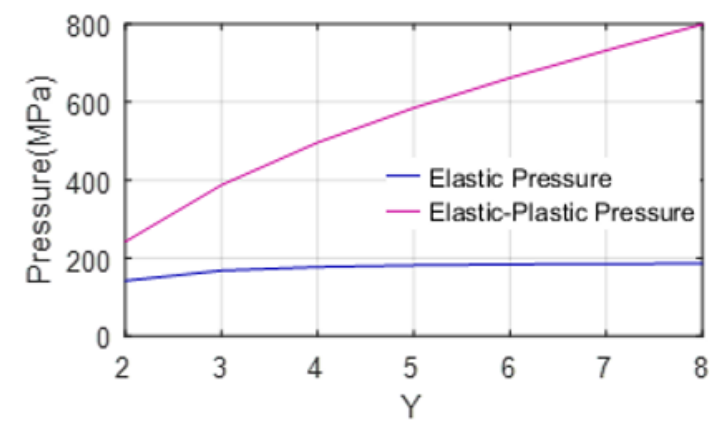

Fig. 4: Pressure over radius ratio

\section{Conclusion}

Stresses in shrink fit consisting of an elastic solid shaft and elastic-plastic hub were computed, using a newly developed model, and their distributions were plotted. A good agreement was found between the developed analytical model and the FE results. The influence of radius ratio on the pressure and interference values was showed.

\section{Funding}

This work was supported by Centre Nationale pour la Recherche Scientifique et Technique in Morocco [Excellence Grant, 78UM52016]

\section{References}

[1] U. Gamer and R. H. Lance, "Residual stress in shrink fits," Int. J. Mech. Sci., vol. 25, no. 7, pp. 465-470, 1983.

[2] U. Gamer and S. Müftü, "On the Elastic-plastic Shrink Fit with Supercritical Interference," ZAMM - J. Appl. Math. Mech. / Zeitschrift für Angew. Math. und Mech., vol. 70, no. 11, pp. 501-507, 1990.

[3] A. A. Burenin, E. P. Dats, and A. V. Tkacheva, "On the modeling of the shrink fit technology," J. Appl. Ind. Math., vol. 8, no. 4, pp. 493-499, 2014.

[4] S. Wang, Q. Tan, Q. Zhuang, and C. Ma, “Analysis and Design of Interference Fit Connection Assembly for Engineering Applications," Icmca, pp. 132-137, 2017.

[5] N. Laghzale and A. Bouzid, "Analytical modelling of elastic-plastic Interference fit joints," Int. Rev. Model. Simulations, vol. 9, no. 3, 2016.

[6] H. J. Kleemola and M. A. Nieminen, "On the strainhardening parameters of metals," Metall. Trans., vol. 5, no. 8, pp. 1863-1866, 1974. 